\title{
Three-dimensional ultrasound image-guided robotic system for accurate microwave coagulation of malignant liver tumours
}

\author{
Jing $\mathrm{Xu}^{1 \# *}$ \\ Zhen-zhong $\mathrm{Jia}^{2 \#}$ \\ Zhang-jun Song ${ }^{3}$ \\ Xiang-dong Yang ${ }^{4}$ \\ Ken Chen ${ }^{4}$ \\ Ping Liang 5 \\ ${ }^{1}$ Department of Electrical and \\ Computer Engineering, Michigan \\ State University, East Lansing, MI, \\ USA \\ ${ }^{2}$ Department of Mechanical \\ Engineering, University of Michigan, \\ Ann Arbor, MI, USA \\ ${ }^{3}$ Shenzhen Institute of Advanced \\ Integration Technology, 1068 \\ Xueyuan Avenue, Shenzhen \\ University Town, Shenzhen, People's \\ Republic of China \\ ${ }^{4}$ Department of Precision Instruments \\ and Mechanology, Tsinghua \\ University, Beijing, People's Republic \\ of China \\ ${ }^{5}$ Department of Ultrasound, The \\ Chinese PLA General Hospital, 28 \\ Fuxing Road, Beijing, People's \\ Republic of China \\ *Correspondence to: Jing Xu, \\ Department of Electrical and \\ Computer Engineering, Michigan \\ State University, East Lansing, MI, \\ 48823, USA. \\ E-mail: xujing0829@gmail.com \\ \# These authors contributed equally \\ to this study.
}

Accepted: 23 March 2010

\begin{abstract}
Background The further application of conventional ultrasound (US) image-guided microwave (MW) ablation of liver cancer is often limited by two-dimensional (2D) imaging, inaccurate needle placement and the resulting skill requirement. The three-dimensional (3D) image-guided robotic-assisted system provides an appealing alternative option, enabling the physician to perform consistent, accurate therapy with improved treatment effectiveness.
\end{abstract}

Methods Our robotic system is constructed by integrating an imaging module, a needle-driven robot, a MW thermal field simulation module, and surgical navigation software in a practical and user-friendly manner. The robot executes precise needle placement based on the 3D model reconstructed from freehand-tracked 2D B-scans. A qualitative slice guidance method for fine registration is introduced to reduce the placement error caused by target motion. By incorporating the 3D MW specific absorption rate (SAR) model into the heat transfer equation, the MW thermal field simulation module determines the MW power level and the coagulation time for improved ablation therapy. Two types of wrists are developed for the robot: a 'remote centre of motion' (RCM) wrist and a non-RCM wrist, which is preferred in real applications.

Results The needle placement accuracies were $<3 \mathrm{~mm}$ for both wrists in the mechanical phantom experiment. The target accuracy for the robot with the RCM wrist was improved to $1.6 \pm 1.0 \mathrm{~mm}$ when real-time $2 \mathrm{D}$ US feedback was used in the artificial-tissue phantom experiment. By using the slice guidance method, the robot with the non-RCM wrist achieved accuracy of $1.8 \pm 0.9 \mathrm{~mm}$ in the ex vivo experiment; even target motion was introduced. In the thermal field experiment, a 5.6\% relative mean error was observed between the experimental coagulated neurosis volume and the simulation result.

Conclusion The proposed robotic system holds promise to enhance the clinical performance of percutaneous MW ablation of malignant liver tumours. Copyright (c) 2010 John Wiley \& Sons, Ltd.

Keywords 3D US; needle placement; microwave coagulation; thermal field

\section{Introduction}

\section{Motivation: ablation of liver tumours}

Liver cancer is the sixth most common type of cancer worldwide, with around 625000 cases recorded in 2002 (1). Approximately half of these 
cases occur in China (1), representing the second deadliest form of cancer in the country (2). The most common malignant liver cancer is hepatocellular carcinoma (HCC) (3), which accounts for $85-90 \%$ of liver cancer cases worldwide (4). There are three main treatment options for liver cancer: (a) surgical resection and in some cases liver transplantation; (b) radiation and chemotherapy; and (c) tumour ablation (5). Only a small portion of patients are candidates for surgical resection, due to the tumour location, underlying liver diseases and additional health problems $(6,7)$. The radiation method is not a curative procedure, although it can shrink the liver tumour. It is still unclear whether chemotherapy increases patient survival (5). The ablative approaches are relatively new treatment options, in which the cancer cells can be directly killed by radiofrequency, freezing or chemical injection via an ablator device inserted into the tumour $(5,6)$. Of all the above methods, image-guided thermal ablation, using energy sources such as radiofrequency (RF), microwave (MW) or laser, may be the most promising treatment option $(6,8)$. Benefits of this minimally invasive procedure include an increased number of patients eligible for potentially curative therapy (6) and reduced morbidity compared with surgery (2).

Conventional thermal ablation is usually conducted in a freehand manner, in which simultaneous manual manipulation of the ablator tool and the ultrasound (US) transducer is often required $(6,7)$. An example is the MW ablative therapy shown in Figure 1. The surgeon mentally relates the real-time $2 \mathrm{D}$ US image with the physical tumour location in the patient's body and then inserts the MW antenna (a needle-type device) via a needle-guiding device attached to the US probe. This needle-guiding device ensures that the antenna-inserting path can always be tracked by the real-time US image. Another surgeon may help to manipulate the US probe to reduce the work load. Once placed at the predetermined

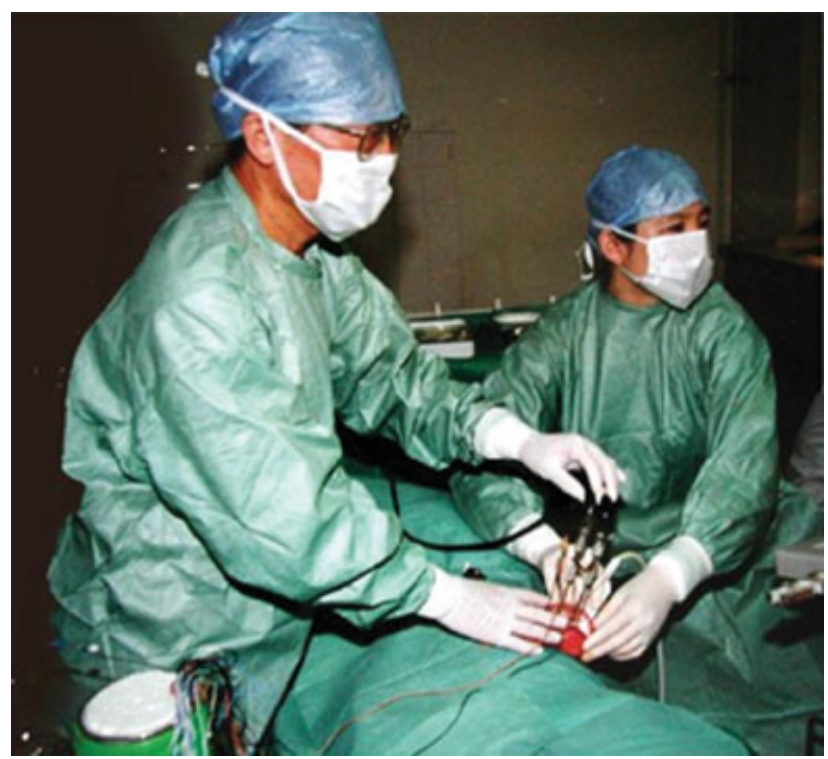

Figure 1. Typical microwave (MW) ablation of liver tumours by 2D ultrasound (2D US) guidance target, the MW antenna can then generate thermal energy to destroy the cancer cells in the tumour. This operational procedure has already been used to treat $>1000$ patients in the Chinese PLA General Hospital (PLAGH) and the results are encouraging (8).

However, the 2D nature of the imaging, inaccurate manual placement of the ablator tool and the resulting skill requirement of the surgeon limit further application of the thermal ablative therapy $(6,9)$. Compared with the manual procedure, the robotic-assisted surgical system is an appealing alternative technique, enabling the surgeon to perform consistent, accurate operation with improved treatment effectiveness $(6,7)$. Moreover, the MW thermal field distribution should be modelled to indentify appropriate MW intensity and coagulation time for the treatment (9), ensuring that the complete coagulated necrosis volume will cover the entire tumour (21). Therefore, a medical robotic system capable of precise needle placement and accurate estimation of the MW thermal field distribution is desired for improved MW ablation of liver tumours (9). However, to the best of our knowledge, such a system is still missing from the market and the literature. Motivated by these facts, we developed an easy-to-use 3D US image-guided robotic system, with knowledge inspired and borrowed from previous research.

\section{Related work}

An important advancement in US image-guided therapy (IGT) is 3D US reconstruction through assembling a series of 2D US images into one 3D volume, either by mechanical, 2D array scanning or free-hand techniques (10). Of all these methods, free-hand tracked 3D US is an appealing approach, allowing flexible operation due to the unconstrained motion of the US probe (11). This method requires spatial tracking of the US probe, usually by an electromagnetic (EM) tracking device, such as the Ascension $\operatorname{Bird}^{\mathrm{TM}}$ position sensor $(6,14)$. This free-hand method has already been realized in software such as Linux-based Stradx $(11,12)$ and the multi-platform open-source package 3D Slicer $(6,13)$ for 3D US reconstruction and visualization. The US probe can also be tracked by other methods, such as the optical positioning system used in the SonoWand ${ }^{\mathrm{TM}}$ system, a commercial 3D intra-operative imaging system combined with navigating capabilities for neurosurgical applications (16). Although most likely resulting in higher spatial accuracy than the EM tracking system (38), the optical positioning system can be inconvenient, since the line-ofsight can be obstructed between the sensor markers and the receiver. Thus, we use the EM tracking method in our robotic system.

The 3D US reconstruction can also be achieved by using robotically positioned US probe information instead of the EM tracker, which is demonstrated by the imageguided robotic system developed for medical diagnostic ultrasound (14). In this application, the measured robot/probe position information was substituted in place 
of the EM tracker data as input to the Stradx program for 3D US reconstruction (14). However, for our application, compared with robotic positioning of the US probe, dexterous manual manipulation is preferred because one objective of the proposed system is minimum modification to the existing manual procedure. Therefore, the EMtracked freehand 3D US method is adopted by our robotic system.

For our application, the most similar research is the free-hand tracked 3D US image-guided robotic system for $\mathrm{RF}$ ablation of liver tumours developed at Johns Hopkins University $(6,7)$. One unique feature of this system is simultaneous real-time tracking of the US probe and the needle-guiding device by the EM tracking system $(6,7)$. In the 'two-handed' configuration, an IBM/JHU LARS robot and a five-degree of freedom (DOF) needledriven robot were used to manipulate the US probe and the needle-guiding device, respectively. A virtual 'remote centre of motion (RCM)' algorithm with an artificial intelligence (AI)-based search optimization was used to control the needle-driven robot (15). It was claimed that precalibration of the robots to the EM tracker was not required, since the motion of both robots was entirely based on the sensed tool location, instead of inverse kinematics (7). By using a reconfigured system with a manually positioned US probe, experiments conducted in (6) indicate that the 3D US -ed robot could eliminate the error bias and thus reduce the invasiveness compared with manual needle insertion. One potential problem with this 'inverse-kinematics-free' approach is the relatively slow motion adjustment, which usually takes $0.8-1.2 \mathrm{~s}$ (15) due to the non-real-time nature of the sensorintegrated searching method. This could be an issue when compensation for the target motions and deformations is desired. Also, it is difficult for this approach to take advantage of already mature robotic techniques, such as servo-control, obstacle avoidance and trajectory planning, where knowledge of the whole robot and the kinematics are often required. In our system, motion control of the robot is based on real-time computation of the inverse kinematics, while spatial tracking of the needle-guiding device is accomplished by the EM sensor or the forward kinematics, based on measured encoder readings.

Some other US image-guided robotic systems have also been reported in the literature. For example, Hong et al. $(17,18)$ developed a motion adaptable needle insertion robot, which consisted of a five-DOF passive arm to place the needle at the skin entry point and a two-DOF needledriving part connected with the US probe (18). By placing the needle in the image plane of the US probe, the needleinsertion path can always be tracked by the US image during the intervention. Then, motion compensation and automatic needle insertion can be achieved via visual servo-control with 2D US image guidance (18). Koizumi et al. developed a non-invasive ultrasound theragnostic system for the destruction of kidney stones by highintensity focused ultrasound (HIFU) (39). In this system, the $3 \mathrm{D}$ relative position between the target and the focus of the HIFU was acquired by two perpendicular US probes. Then, the focused position of the HIFU was commanded to track and follow the target by feedforward control, based on the quasi-periodical motion of the kidney. Compared with the robotically positioned US probe in these two examples, free-hand tracked 3D US is used by our system for more dexterous and flexible operation.

While the 3D US image-guided robotic system helps to place the ablator tool into the centre of the liver tumour accurately, precise estimation of the 3D thermal field distribution is also needed to identify the MW power and coagulation time for the ablation. This ensures that complete coagulated necrosis volume can cover the entire tumour (21), with minimum invasiveness to normal tissues. Compared with the popular topic of RF thermal field analyses $(19,20), 3 D$ thermal field modelling for the MW ablation of liver tumours receives less attention. One of the few exceptions is the dynamic simulation model (21) proposed by the PLAGH group for the manual MW ablation procedure. However, the MW thermal energy specific absorption rate (SAR) distributions were simply established by curve-fitting of experimental data, while a general SAR model was missing in this model. Also, the dynamic 3D temperature distributions were reconstructed with finite-element method (FEM) using ANSYS software (21). This approach creates a technological and logistical challenge to integrate thermal modelling into the surgical navigation system, due to the non-open commercial software. In this paper, we propose a method for real-time 3D modelling of the MW thermal field distribution, which can be integrated with the 3D US imaging module for MW ablation therapy. In our model, the semi-empirical formula of the SAR model from (33) is modified by assuming a rotationally symmetrical distribution about the central axis of the MW antenna. Then, the dynamic 3D temperature distribution can be identified by plugging the SAR model into the heat transfer equation.

Intended for precise needle placement and accurate 3D MW thermal field estimation, we constructed the 3D US image-guided robotic system for MW ablation of liver tumours by integrating, in a safe, practical and easy-to-use manner: (a) real-time tracking of the US transducer and the needle; (b) 3D US reconstruction and visualization; (c) a medical robot manipulating the needle; and (d) a MW thermal field simulation module. In our system, spatial tracking of the US probe is accomplished by the EM tracking device. The needle can be tracked by either the EM tracker or the measured robot encoder information. All these modules, including the free-hand tracked 3D US and robot control, are integrated into self-developed surgical navigation software. It should be noted that our robotic system can be easily reconfigured for other applications, although it is initially designed for the MW ablation of liver tumours. In the following sections, we briefly outline key components of the system. 


\section{Materials and Methods}

Our robotic system consists of four basic components: (a) an imaging module for 3D US reconstruction, slice navigation, and visualization; (b) a five-DOF medical robot to manipulate the needle (MW antenna); (c) a MW thermal field simulation module; and (d) surgical navigation software which integrates the imaging, thermal field simulation and supervisory control of the robot. We developed all these components by using commercially available parts, such as the EM device and some open source packages. These components will be described below after discussing the overall architecture and the operation procedure of the proposed robotic system.

\section{System architecture and operation procedure}

Figure 2 illustrates a typical clinical room set-up and the schematic architecture of our robotic system, which was finalized after intensive field study and discussion with physicians. A five-DOF needle-driven robot with a needleguiding device as its end-effecter is used to position the needle. The real-time spatial coordinates of the manually positioned (by surgeon 1) US probe, which is required for the imaging module, can be traced by an EM tracking system (Flock of Birds, Model 6D, Ascension Technology Inc.). The root-mean-square (RMS) value of the static positional and angular accuracy for the EM sensor is $0.18 \mathrm{~mm}$ and $0.5^{\circ}$, respectively (22). The coordinate system determined by the EM transmitter, which is rigidly connected to the robot base, is considered as the world coordinate throughout this paper. The coordinate of the needle can be traced either by the EM tracker (through EM receiver 2) or the robot through the encoder reading of each joint. A PC-based surgical workstation hosts the surgical navigation software, including the US imaging, thermal field simulation and supervisory control of the robot. Once the needle-guiding device has been robotically manipulated to the desired position, another physician (surgeon 2) could manually insert the needle to the target location under the guidance of US images. Then, the thermal energy generated by the MW machine can be used to coagulate the liver tumours.

Figure 3 shows a flowchart of a typical operation procedure for MW ablation therapy, using our image-guided intervention system. The proposed procedure follows the general framework (23) of computer-integrated surgery (CIS) and consists of three stages: (a) preoperative modelling and surgical planning; (b) intraoperative therapy; and (c) postoperative assessment. In the following, based on Figures 2 and 3, we give a more detailed procedure, enabling the robotic system to execute accurate MW ablation.

In the preoperative stage, a 3D model of the liver, including the tumours, can be reconstructed from a group of manually scanned (by surgeon 1 in Figure 2)

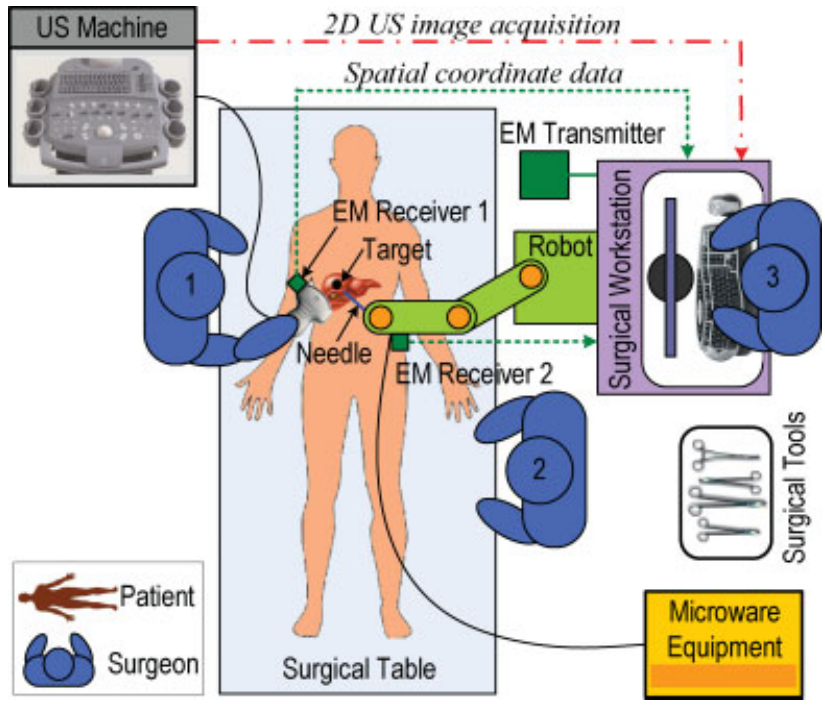

Figure 2. Schematic illustration of the proposed image-guided robotic system for the MW ablation therapy. A freehand EM-tracked US probe is used for the US imaging module, while a medical robot is used to manipulate the needle. Spatial tracking of the needle can be accomplished by either the EM tracker or the measured robot position information

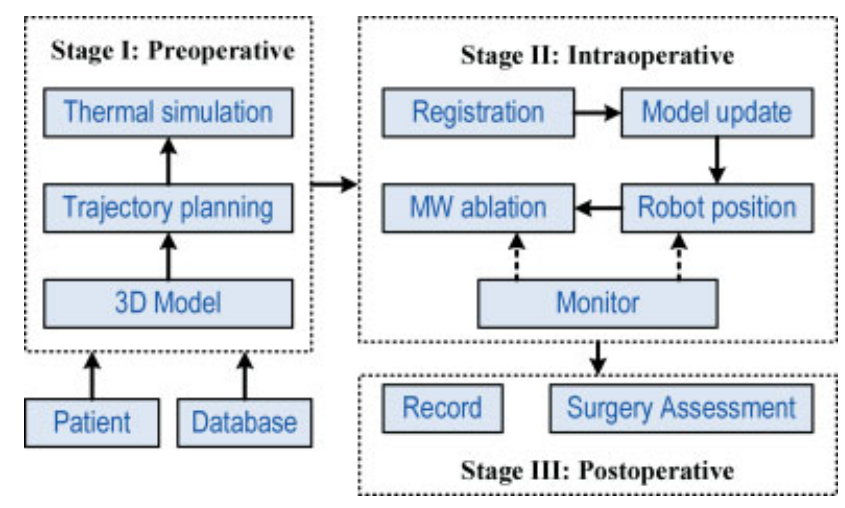

Figure 3. Typical robotic-assisted operation procedure for MW ablation therapy

2D US images by the imaging module. According to the lesion location in the reconstructed 3D model, a safe needle-insertion path can be determined to avoid the blood vessels and ribs by using robot trajectory planning. Then, the thermal field simulation module can be used to identify an appropriate MW power level and coagulation time required to destroy the liver tumours. Sometimes multiple coagulations may be required for the ablation.

In the intraoperative stage, the specific patient model and planned trajectory is registered to the patient, based on markers in the patient's body surface and the blood vessels in the liver (24). The planned trajectory can be adjusted accordingly for a safe needle-insertion path. Then, the needle can be moved to the entry point and aligned with the entry vector by the robot through inverse kinematics. A physician (surgeon 2 in Figure 2) then inserts the needle into the target using US imaging guidance. Once the needle has been placed into the 
desired position, the surgeon can turn on the MW machine for thermal ablation.

It should be noted that inevitable motions due to respiratory function and some unexpected movements will affect the accuracy of registration. Therefore, fine registration is desired before needle insertion. In this paper, we propose a method called 'slice guidance' to reduce the error caused by respiratory motion, by comparing the real-time US and the corresponding resliced image from the original 2D scans. Implementation of this slice-guidance method will be discussed further in subsequent sections.

After the operation, the surgical information and postoperative results are collected to verify that the surgical plan has been successfully executed and to evaluate the effectiveness of the operation. All the information is then recorded for long-term tracking of the clinical results and evaluation of the proposed method.

As shown in Figure 2, the proposed robotic system is realized by adding an EM tracking system, a needledriven robot, and a surgical workstation to the existing manual operation procedure (Figure 1). With a properly designed human-machine interface, such a configuration will not introduce complex engineering problems into an otherwise rather straightforward MW ablation procedure. Moreover, the accuracy and consistency of MW ablation therapy can be improved by the coupling of thermal simulation, US imaging, patient-specific models and robotic-assisted needle placement.

\section{Freehand tracked US imaging module}

The freehand tracked US imaging module enables 3D US reconstruction, slice guidance and visualization for surgical planning and intraoperative registration. As shown in Figure 4, the module consists of: (a) a conventional 2D US machine; (b) a PC-based surgical workstation; and (c) an EM tracking system, recording the position and orientation of the US probe and thus tracking the spatial coordinates of each $2 \mathrm{D}$ scan in a real-time manner. Image acquisition is achieved by the workstation via a video frame grabber card connected to the video output port of the US machine. The main components of the imaging module are:

- Data acquisition, which simultaneously captures the 2D US images and the corresponding spatial coordinates of each scan in the EM transmitter coordinate frame (also the world frame in this paper).

- $3 D$ US reconstruction, which converts the acquired $2 \mathrm{D}$ images into a structured 3D volume. This can be done by a user-selected interpolation algorithm with options including: voxel nearest neighbour (VNN), pixel nearest neighbour (PNN) and distance-weighted (DW) methods (26). Compared with the VNN method, the DW interpolation could reduce the misalignment of the B-scans caused by registration errors. Meanwhile, both the bin-filling stage and the hole-filling stage in the PNN

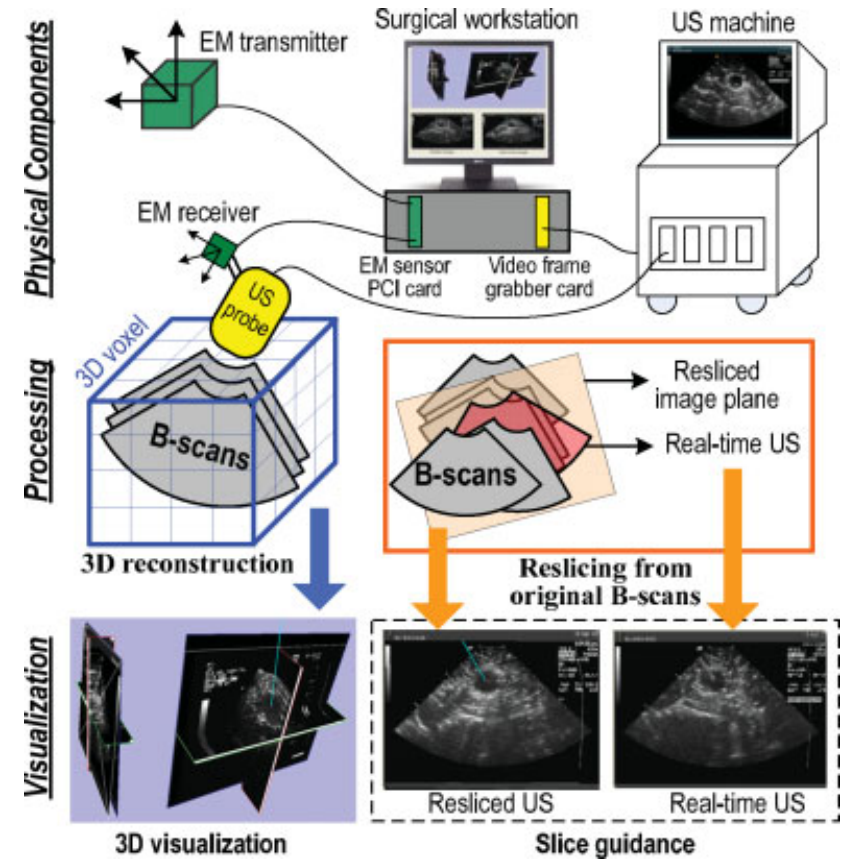

Figure 4. Freehand tracked US imaging module

method will introduce errors to the final result $(38,24)$. Therefore, the DW method is selected by our system for precise 3D reconstruction $(24,26)$.

- Feature segmentation, which extracts the boundary of the region of interest (ROI), such as the clinical tumour and the portal vessels in the liver. We apply an adaptive level-set method (27) to the structured arrays of 2D images resliced from the reconstructed 3D volume. After manual selection of the lesion boundary in one or several reconstructed images, the remaining images can be automatically segmented by the improved level-set method with the results of neighbouring images as the initial boundary (27). These automatically extracted boundaries can be further refined manually by the surgeon.

- Visualization, in which the reconstructed 3D dataset and the ROI boundaries are visualized by volume-rendering and surface-rendering techniques, respectively (24). Using these techniques, the ROI boundary can be emphasized in the volume-rendered 3D model, resulting in a better visualization effect.

- Slice guidance, which is intended for fine registration for intraoperative intervention after the registration by body surface markers and portal vessels in the liver (24). Based on the real-time spatial coordinates of the EM tracker, corresponding images can be resliced from the original B-scans $(24,25)$ by using an interpolation algorithm. The resliced image will match the realtime image captured from the US machine if the coordinates of the reconstructed model agree with the actual tumour location. If not, the patient will be asked to regulate his breathing and thus adjust the tumour location until the resliced image matches the real-time US. A fine registration can usually be achieved by repeating the above process two or three times (24). 
- Off-line calibration, which determines the transformation matrix between the US image frame and the EM receiver coordinate system. Then each $2 \mathrm{D}$ scan can be converted into the world coordinate system, based on the identified transformation matrix. The fast calibration method (28) proposed by Pagoulatos is modified for our application (24).

\section{Needle-driven robot design and control}

While meeting the safety, reliability and positioning accuracy requirements, appropriate configuration is required for the robot to ensure proper functioning of the EM tracking system. The reason is that the accuracy of the needle placement highly depends on the EM tracking system (6), whose accuracy may be affected by the driving motor and ultrasound machine. Our assessment experiment (24) of the EM sensor indicates that the US machine, MW machine, driving motor and surgical workstation will affect the reading of the EM sensor, and thus should be placed relatively far away from the tracking system. Additionally, the surgical scalpel, US probe and ablation needle can be freely placed, since they do not affect the EM sensor reading (24). In addition, convenient human-machine interaction is also desired for practical and comfortable operations.

The SCARA mechanism has relatively high positioning accuracy due to the decoupled motion in the horizontal and vertical direction. In addition, it allows comfortable human-robot interaction and provides enough operating space for the physician. Therefore, we developed our robot based on the SCARA configuration, as shown in Figure 5. In addition, two types of wrists were developed: (a) an RCM wrist, consisting of a mechanically constrained RCM mechanism; and later (b) a non-RCM wrist, consisting of two rotational joints. The RCM mechanism may be complex and expensive to produce (15) because of the precise manufacturing and assembly requirements. Thus, the non-RCM wrist is preferred in real applications. For the wrists shown in Figure 5, the wrist joint one has a range of $270^{\circ}$ about its axis of rotation (vertical direction), and wrist joint two has a range of $150^{\circ}$ about its axis of rotation (horizontal direction). Thus, both wrists permit large orientation angle adjustment in a relatively small space. In order not to affect the EM sensor reading, the robot is made of magnetic-free stainless steel, while all the driving motors in the robot are placed far away from the EM transmitter (at least $400 \mathrm{~mm}$ in the vertical direction). For simplification, in subsequent sections, as appropriate, the term 'RCM robot' will be used to indicate the needledriven robot installed with the RCM wrist, while the term 'non-RCM robot' indicates the needle-driven robot installed with the non-RCM wrist.

Reliability and safety must be ensured for the needledriven robot because the robotic-assisted operation involves intensive human-robot interactions. Besides conventional methods such as communication reliability design, limit switch, and emergency stop, some other
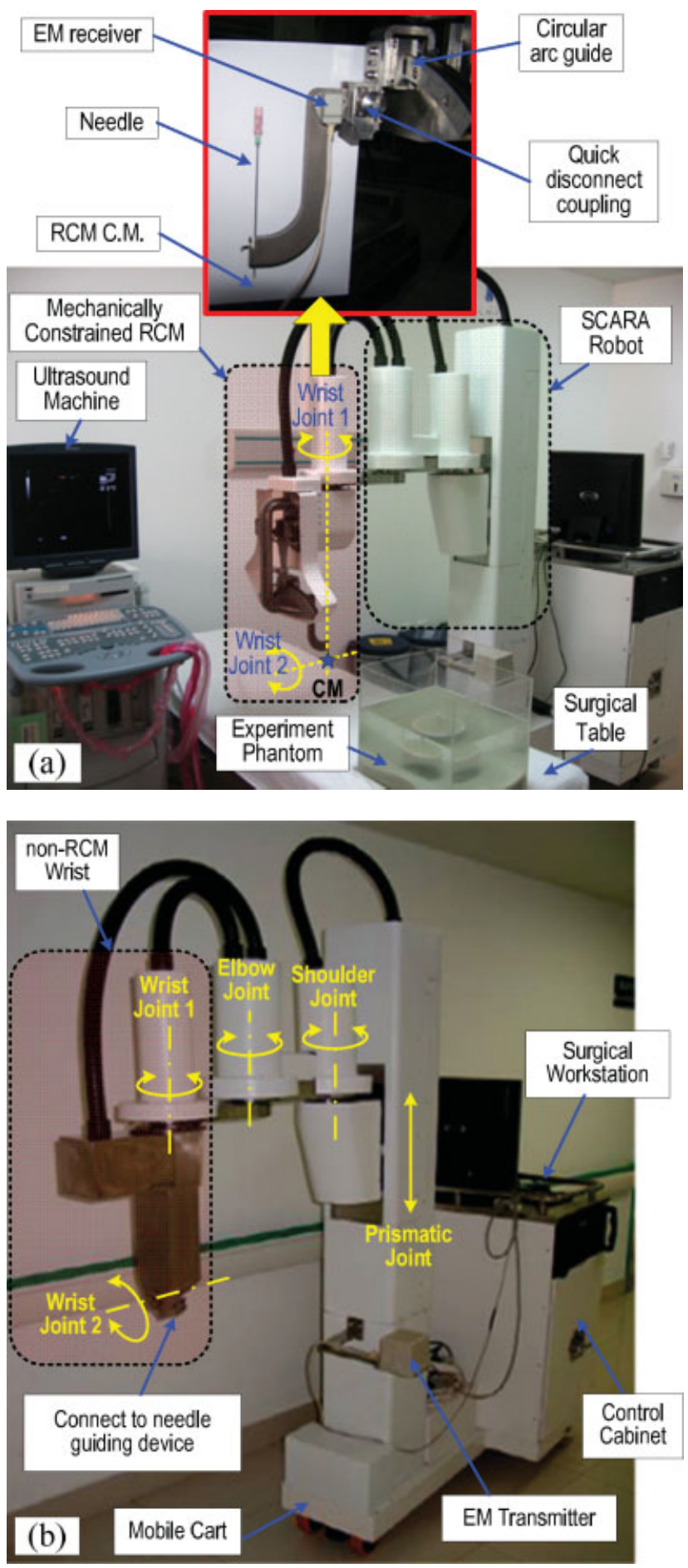

Figure 5. (a) Needle-driven robot with a mechanically constrained RCM wrist; (b) needle-driven robot with a non-RCM wrist. In (a), the centre of motion (C.M.) for the RCM wrist is simply the intersection of the wrist joints' axes of rotation

techniques have also been used to improve the above requirements of the robot for clinical applications:

- The robot can be freely switched between two modes: the active mode and the passive mode. The robot works in active mode for needle placement under normal conditions. Once an emergency occurs or the operation 
is finished, the robot can be switched to passive mode and moved away from the patient quickly.

- The robot can resist a $5 \mathrm{~kg}$ impact, eliminating the influence of unexpected impacts on the needle placement during the operation.

- The needle-guiding device is connected to the robot through a quick-disconnect coupling. This 'quick release' design enables easy and reliable sterilization of the guiding device.

- The robot is mounted on a mobile cart, enabling a quick and flexible clinical room set-up. The mobile cart can be lifted and then locked by step motors to avoid unexpected motion of the robot base during the operation.

Errors in the design, fabrication and assembly process will affect the positioning accuracy of the robot, which can be partially compensated by robot calibration techniques. The non-RCM robot achieves a repetitive positioning accuracy of $0.06 \pm 0.03 \mathrm{~mm}$ and an absolute positioning accuracy of $0.69 \pm 0.34 \mathrm{~mm}$ after calibration and compensation by a CimCore ${ }^{\mathrm{TM}}$ Infinite 2.0 (31) portable coordinate measure machine (CMM). Therefore, reliable real-time spatial tracking of the needle-guiding device can be achieved by the robot through optical encoder readings of each joint. It should be pointed out that the optical encoder reading for wrist joint two in the RCM robot can be substituted by the EM receiver (Figure 5) attached to the needle-guiding device, to eliminate the error introduced by the mechanical transmission.

In our system, the EM transmitter is rigidly attached to the robot base. To integrate the freehand-tracked US imaging module and the surgical robot module, we need to identify the spatial relationship between the coordinate frame of the robot base and the EM transmitter frame (also the world frame in this paper). This can be done by using the robot calibration technique with a standard 3D template. Coordinates of the marked points in the 3D template can be calculated by robot forward kinematics, based on the sensor readings of each joint. By using methods in $(29,30)$, we can compute the transformation matrix between the 3D coordinates of these markers in these two coordinate frames. In our non-RCM robotic system, 75 markers are selected in the 3D template for the calibration. The resulting positioning error for coordinate mapping was $0.81 \pm 0.53 \mathrm{~mm}$.

Due to the decoupled configuration, a closed-form solution of the inverse kinematics for both robot configurations can be derived by using the geometrical method given in (32). Then, motion control of the robot, including the real-time motion compensation (18), can be achieved via real-time computation of inverse kinematics. In practical applications, a simple robot motion-planning (SRMP) method for needle placement can be outlined as: (a) determine the desired joint angles according to the target location and the needle entry vector; (b) set an appropriate value for the prismatic joint, ensuring a safe distance from the robot to the patient in the vertical direction; (c) command all the rotational joints to their desired angles; (d) move the prismatic joint downwards until reaching the predefined position for needle placement.

\section{MW thermal field modelling}

The MW thermal field modelling module is used to identify the MW power level and the coagulation time needed for the ablation, ensuring that the complete coagulated necrosis volume can cover the entire tumour (21). Inappropriate selection of the parameter values may lead to unintended or severe consequences - overdestruction of the normal tissues or leaving unkilled cancer cells in the tumour. The modelling process generally involves the determination of the specific absorption rate (SAR) of the thermal energy in the tissue and the identification of the temperature field distribution by heat transfer equations $(21,33)$. Zhu et al. (33) proposed a semi-empirical formula to identify the 3D SAR distribution of the MW energy, based on experimental data from tissue microwave-equivalent phantom gel. In our model, by assuming a rotationally symmetrical thermal field distribution about the central axis of the MW antenna, and thus eliminating the angular dependence in the original equation given in (33), the 3D SAR distribution can be expressed in the MW antenna frame as:

$$
S A R(r, z)=C_{\mathrm{t}} W \frac{[2 \varepsilon r+(N-2)] e^{-2 \varepsilon r}}{r^{N}} e^{-z^{2} / z_{0}^{2}}
$$

where $W$ is the MW power, $C_{t}$ is a scale factor, $\varepsilon$ is an attenuation constant for the tissue, $N$ is an exponential constant, which depends on the MW antenna, $r$ is the radial distance from the centre of the antenna, $z$ is the axial distance from the electrode tip and $z_{0}$ is the axial decay distance defined in (33). Extensive experiments are required to identify these parameter values, which usually depend on the tissue and thermotherapy conditions (9).

The MW thermal energy generated at a given coordinate, which is proportional to the corresponding SAR value, is considered as a thermal source in the heat transfer equation. Then, also by considering the cooling effect of blood circulation, the dynamic 3D temperature distribution in the tissue can be identified by the following heat-transfer equation (40):

$$
\rho c \frac{\partial T}{\partial t}=\kappa \nabla^{2} T-\rho \rho_{b} c_{b} F\left(T-T_{b}\right)+\rho \cdot S A R
$$

where $T$ is the temperature, $t$ is the time, $\rho$ is the density of the tissue, $c$ is the specific heat capacity of the tissue, $k$ is the thermal conductivity of the tissue, $\rho_{b}$ is the density of the blood, $c_{b}$ is the specific heat capacity of the blood, $T_{b}$ is the temperature of the blood and $F$ is the blood flow rate.

For visualization purposes, we convert the temperature field distribution from the above cylindrical frame into 


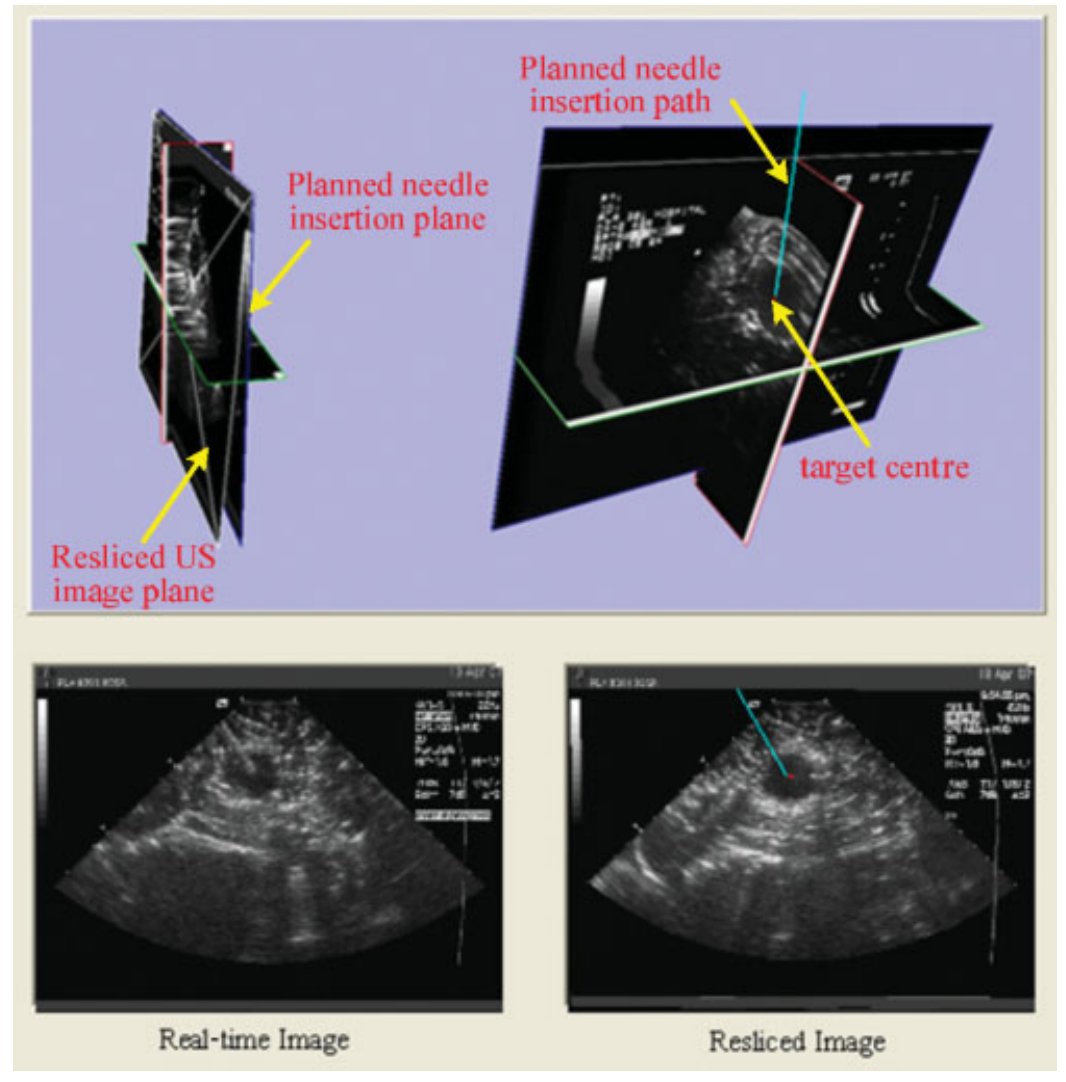

Figure 6. One snapshot of the surgical navigation software with annotations added to illustrate the 'slice guidance' method

a 3D Cartesian coordinate system. Then, the thermal field distribution in the Cartesian frame can be calculated by the finite-difference time-domain (FDTD) method. The initial temperature of the tissue is set to $37^{\circ} \mathrm{C}$. In addition, the temperatures of thick blood vessels and the abdominal cavity are also set to $37^{\circ} \mathrm{C}$ during the calculation to simulate the cooling effect.

\section{Surgical navigation software}

The surgical navigation software integrates the US imaging module, the MW thermal field estimation module, and the robot supervisory control in a practical and easy-to-use manner, enabling convenient and reliable human-machine interaction. This navigation software was developed by using Microsoft Visual $\mathrm{C}++$, based on the Windows operating system. Some open-source software packages, such as VTK [Visualization Toolkit (36)] and ITK [Segmentation and Registration Toolkit (37)] were used for medical image processing and 3D reconstruction (24). Figure 6 shows one snapshot of the surgical navigation software during intraoperative intervention.

\section{Experiments, Results and Discussion}

In order to verify and determine the positioning accuracy of our robotic system, we conducted a series of needleplacement experiments, using a mechanical phantom, an artificial-tissue phantom and an ex vivo test on bovine liver (24). We used an 18-G needle $(0.84 \mathrm{~mm}$ i.d., $1.27 \mathrm{~mm}$ o.d.) for all these needle-placement experiments. Also, another mechanical phantom based on a 3D template was used to identify the accuracy of 3D US reconstruction and the contribution of the US imaging module to the positioning error of the overall system. Besides these accuracy tests, we also conducted an ex vivo experiment on porcine liver to verify the proposed model for MW thermal field simulation.

\section{Mechanical phantom experiment}

The objective of the mechanical phantom experiment was to identify the robotic positioning accuracy in a simulated clinical environment from an engineering perspective. As shown in Figure 7, the mechanical phantom we used was a transparent plastic tank filled with water. The tips of two $1.5 \mathrm{~mm}$ diameter plastic pins were used to simulate the needle entry point and the target centre for needle placement. A bifurcated vessel model, which was fabricated by filling molten agarose into an injection mould, was used to simulate the hepatic portal vein for coarse registration in the intraoperative stage. The plastic pins and the vessel model were fixed onto a plastic plate immersed in water. In order to eliminate the impact introduced by ultrasound reflection, the bottom of the water tank was filled with a $7 \mathrm{~mm}$ thick layer of fine sand to absorb the ultrasound wave. 


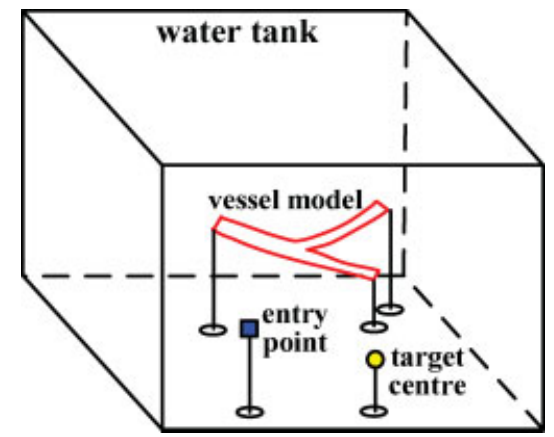

Figure 7. Mechanical phantom for robotic needle placement experiment

The experimental procedure follows the aforementioned general workflow of the robotic system and is outlined as follows (24):

1. Data acquisition. Manually scan the phantom by a translational sweep with the US probe perpendicular to the bottom of the tank, while recording the 2D US images and the corresponding EM tracker data. About 100 US images are acquired, and the distance between two consecutive images is about $2 \mathrm{~mm}$.

2. $3 D$ reconstruction and segmentation. Reconstruct the $3 \mathrm{D}$ US model of the scanned volume and extract the corresponding boundaries of ROIs, such as the needle entry point, target centre and the vessel model, using the method described above.

3. Surgical planning. Interactively select the needle entry point and the target centre for needle placement in the surgical planning software. Due to US imaging artifacts, the tips of the plastic pins are displayed as bright regions in the US image. The geometric centres of these bright regions, which can be automatically calculated by the planning software, are considered as the needle entry point and target centre.

4. Registration \& slice guidance. Determine the transformation matrix between the preoperative vessel and intraoperative vessel models by an ICP algorithm $(34,35)$ and then register the reconstructed 3D model and the planned trajectory to the actual phantom. Fine registration can be achieved by using the sliceguidance method described above. If the phantom is fixed during the experiment, the registration step can be skipped, since the transformation matrix is simply an identity matrix.

5. Robot motion. Command the robot to the desired position, by using the SRMP method described above.

6. Needle insertion. Manually insert the needle via the needle-guiding device. The needle insertion depth can be monitored by the depth marker or the EM tracker attached to the needle (Figure 8).

7. Assessment. Use calipers to measure the needle insertion accuracy. As shown in Figure 9, the entry accuracy (or needle-entering accuracy), $d_{e}$, is defined as the distance between the entry plastic pin tip and the axial centre of the needle, while the target accuracy (or needle-targeting accuracy), $d_{t}$, is defined as the

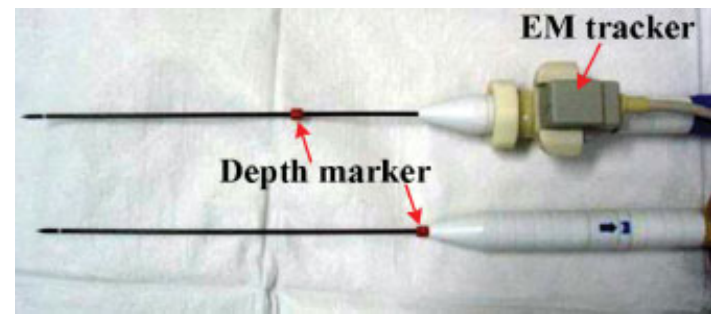

Figure 8. Needle insertion depth marker and EM tracker

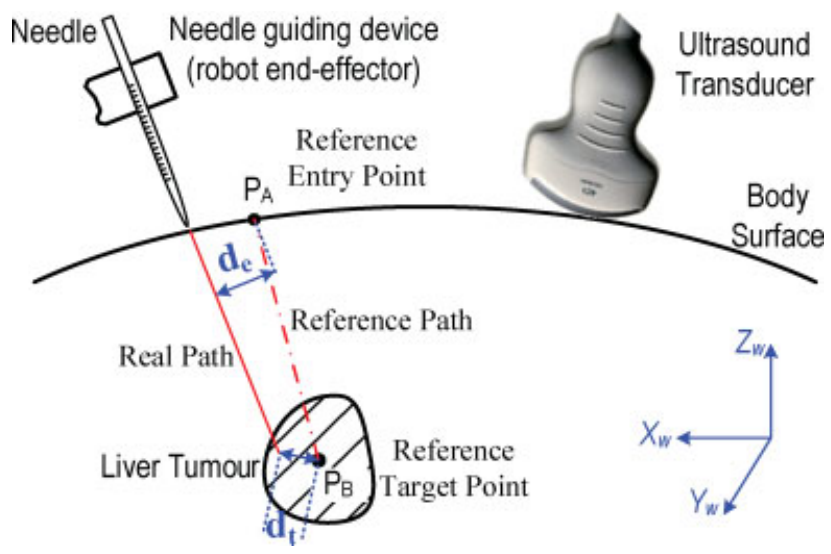

Figure 9. Robotic-assisted needle placement

Table 1. Positioning accuracy of the robotic system

\begin{tabular}{llc}
\hline Accuracy/system & RCM robot & Non-RCM robot \\
\hline$d_{e}(\mathrm{~mm})$ & $2.62 \pm 1.21$ & $2.01 \pm 0.73$ \\
$d_{t}(\mathrm{~mm})$ & $2.94 \pm 1.34$ & $2.63 \pm 1.20$ \\
\hline
\end{tabular}

distance between the target plastic pin tip and the inserted needle tip.

By fixing the mechanical phantom and thus skipping the registration step, the above procedure was repeated 30 times. The experimental results are listed in Table 1. Both the RCM robot and the non-RCM robot achieved very high needle-placement accuracy for fixed rigid targets (plastic pins). It should be mentioned that the RCM robot achieved relatively lower accuracy than the non-RCM robot, mainly due to fabrication and assembly errors in the mechanically-constrained RCM mechanism.

In order to identify the contribution of the imaging module to the overall positioning accuracy, we tested the 3D US reconstruction accuracy by phantom experiment of a 3D template consisting of 10 plastic pins (Figure 10). We first manually scanned the 3D template by a translational sweep with the US probe perpendicular to the inclined surface of the template, while acquiring the 2D US images and corresponding tracker data. The distance between two successive 2D US images was about $1 \mathrm{~mm}$. After the data acquisition, we reconstructed the $3 \mathrm{D}$ model 


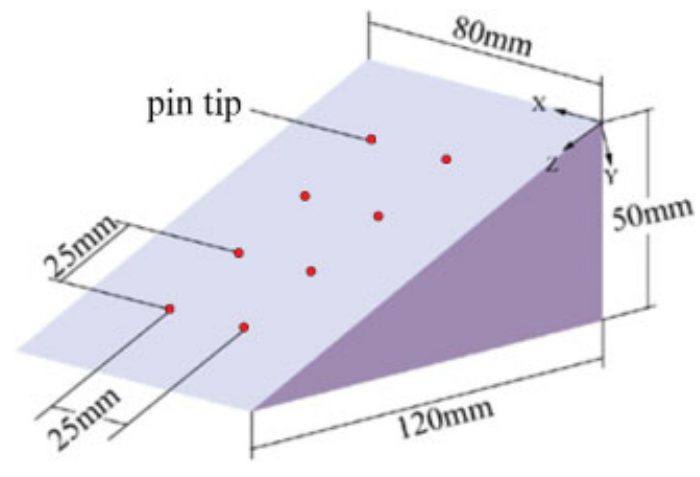

Figure 10. 3D template consisted of 10 plastic pins, $1.5 \mathrm{~mm}$ in diameter

of the template and then converted the model from the EM transmitter frame into the template frame. Let $\left(x_{i}, y_{i}, z_{i}\right)$ and $\left(x_{i}{ }^{\prime}, y_{i}{ }^{\prime}, z_{i}{ }^{\prime}\right)$ denote the original and the reconstructed coordinate of the ith plastic pin tip in the template frame. The reconstruction errors along the $x, y$ and $z$ axes can be defined as $\Delta x=\operatorname{avg}\left(\left|x_{i}-x_{i}^{\prime}\right|\right)$, $\Delta y=\operatorname{avg}\left(\left|y_{i}-y_{i}^{\prime}\right|\right)$ and $\Delta z=\operatorname{avg}\left(\left|z_{i}-z_{i}{ }^{\prime}\right|\right)$, respectively. The calculated reconstruction errors along the $x$ and $y$ axes were $0.28 \mathrm{~mm}$ and $0.30 \mathrm{~mm}$, respectively, which were almost the same as the resolution of the 2D US images. The reconstruction error along the $z$ axis was $0.61 \mathrm{~mm}$, which was greater than the $x$ and $y$ axis errors, due to the non-ideal tracking of the EM sensor (22), floating point truncation (1-2 pixels) during image processing, etc.

By using the EM-tracked needle to identify the coordinates of two plastic pins, and thus eliminating the error of the US imaging module in the previous mechanical phantom experiment, we tested the positioning accuracy of the robot without image guidance. The entry and target errors for the non-RCM robot, excluding those introduced by the US imaging module, were $1.17 \pm 0.82 \mathrm{~mm}$ and $1.40 \pm 0.84 \mathrm{~mm}$, respectively. We see that the overall positioning error for the needle placement almost equals the summation of errors introduced by the US imaging module and the needle-driven robot.

\section{Artificial-tissue phantom experiment}

The artificial-tissue phantom consisted of materials with different tissue attenuation coefficients to simulate the liver tumour and normal liver tissue for US imageguided therapy. This artificial tissue phantom, as shown in Figure 11, was fabricated by the Institute of Acoustics at the Chinese Academy of Science. The whole surface of the phantom container was covered with Plexiglas except the acoustic window at the top of the container, which was composed of polyester film. The phantom container was filled with tissue-mimicking (TM) phantom material to simulate the normal tissue. It was displayed as the background in the US image. A series of $1 \mathrm{~cm}$ diameter spheres were embedded in the TM phantom material to simulate clinical liver tumours. The tissue attenuation coefficients for the TM phantom material and

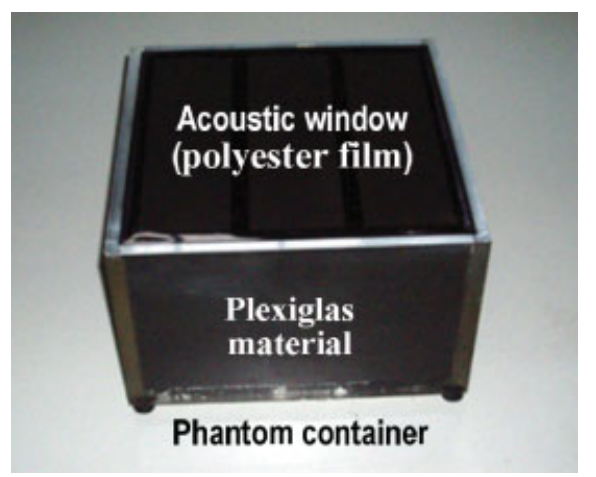

Figure 11. Artificial tissue phantom for US image-guided needle placement

the tumour-mimicking spheres were $0.7 \mathrm{~dB} / \mathrm{cm} \cdot \mathrm{MHz}$ and $0.35 \mathrm{~dB} / \mathrm{cm} \cdot \mathrm{MHz}$, respectively. Both of them had the sonic velocity of $1540 \pm 10 \mathrm{~m} / \mathrm{s}$.

The experimental procedure can be outlined as follows: (a) volume scan and data acquisition; (b) 3D US reconstruction and feature segmentation of the artificial tumour; (c) identification of the target, i.e. the geometrical centre of the artificial tumour; (d) robot motion; (e) needle insertion with real-time US monitoring of the inserting depth; and (f) measuring the needle insertion accuracy from a 2D US image captured by the US machine after the insertion. The above procedure was repeated 40 times in the RCM robot. The resulting target error was $1.6 \pm 1.0 \mathrm{~mm}$. One reason for this improved target accuracy compared with the previous mechanical phantom experiment should be ascribed to the fine adjustment of the needle by the surgeon through real-time US feedback.

It should be mentioned that the needle (PCT-B 18-G) may be curved due to the shearing force exerted by the TM material during the insertion, resulting in missing of some deep targets, as shown in Figure 12. The distortion can be significantly reduced by improving the rigidity of the needle.

\section{Ex vivo experiment}

We also tested the accuracy of needle placement by using an ex vivo experiment of bovine liver (Figure 13) with an embedded $2 \mathrm{~cm}$ diameter artificial tumour, which was fabricated by filling molten agarose into a spherical injection mould. One objective of the bovine liver experiment is to evaluate the slice guidance method, which is intended to reduce the impact of target motion (mainly introduced by respiratory motion in real surgery) on the accuracy of needle placement.

The preoperative procedure was similar to the artificialtissue experiment. During the intraoperative stage, the US probe was used to exert some pressure onto the bovine liver, resulting in the deformation and motion of the liver. This would cause motion of the target tumour embedded in the bovine liver. In order to evaluate the slice guidance method, we conducted the experiments under 


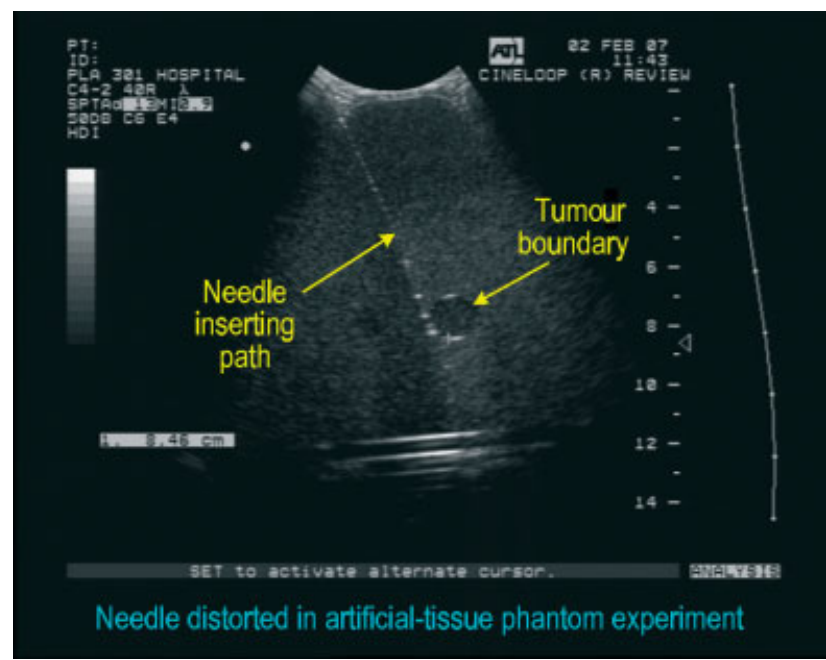

Figure 12. An 18-G needle was curved in the artificial-tissue phantom experiment, resulting in missing of the target at a depth of about $84 \mathrm{~mm}$
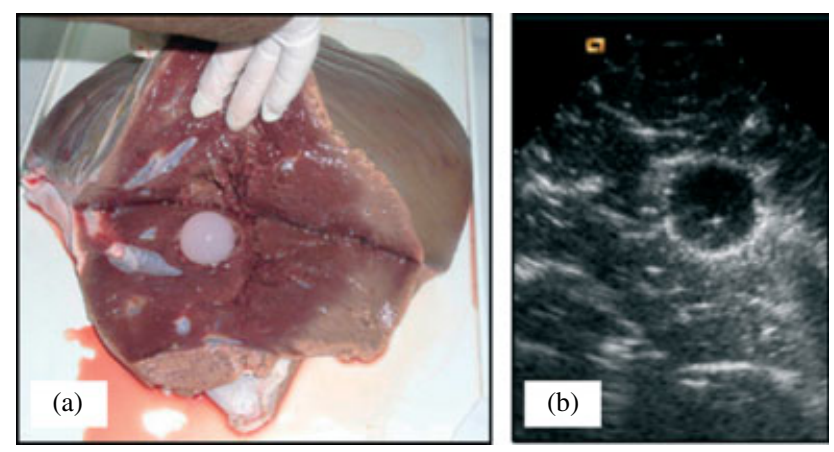

Figure 13. Bovine liver with an embedded artificial tumour: (a) set-up; (b) 2D US image

two scenarios: (a) adjust the position and magnitude of the pressure exerted by the US probe such that the resliced image matches the real-time US image, and insert the needle; (b) insert the needle even when the resliced image and the real-time US image do not match. Figure 6 gives one snapshot of surgical navigation software for scenario (a). Corresponding experimental results are given in Figure 14 by cutting the artificial tumour along the needle inserting path after needle placement.

From the above experiment, we see that the accuracy of needle placement will be reduced due to the target motion caused by uneven pressure exerted by the US probe during the intraoperative intervention. The proposed slice guidance method can be used to reduce this error, resulting in improved accuracy of needle placement. Based on the slice guidance method, we tested the needle placement accuracy of the non-RCM robotic system by using a procedure similar to the artificialtissue experiment. The target error was $1.8 \pm 0.9 \mathrm{~mm}$. This is relatively less than that of mechanical phantom experiment, due to the improved registration between the real target and the preoperative model by fine adjustment of the target location according to real-time US feedback and the slice guidance method.
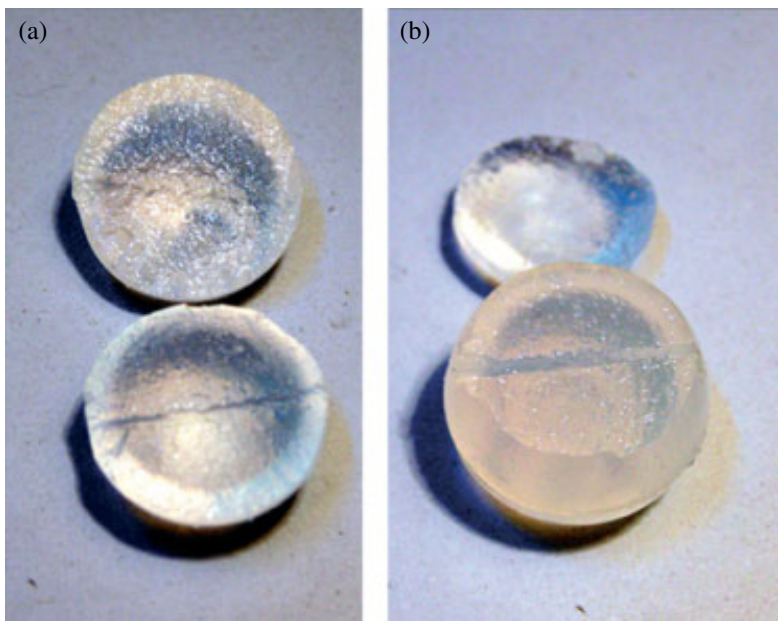

Figure 14. The ex vivo experimental results corresponding to scenarios (a) and (b)

\section{Thermal field estimation experiment}

In order to verify the MW thermal field simulation model, especially its performance in predicting the complete coagulated neurosis volume, given MW power level and coagulation time, we conducted an experiment on ex vivo liver tissues. Porcine liver was selected, due to similar thermal conductive properties to the human liver in clinical applications. In order to obtain a large thermal field, we selected liver tissue with thickness of $>5 \mathrm{~cm}$ (21). The MW machine used in the experiment was the UMC-1 MW generator (The 207 Institute of Aerospace Science and Technology Corporation, Beijing, China) with an operating frequency of $2450 \mathrm{MHz}$ and output power in the range $10-80 \mathrm{~W}$. The MW electrode was $1.4 \mathrm{~mm}$ in diameter and had a $27 \mathrm{~mm}$ naked tip for heat generation. The temperature distributions were obtained by thermocouples (resolution $0.1^{\circ} \mathrm{C}$ ) inserted in the porcine liver tissue.

The geometrical grid size we selected for the MW thermal field simulation was $1.5 \mathrm{~mm}$. The temperature of each voxel in the thermal field was calculated by the FDTD method, with an iterative time span of $5 \mathrm{~s}$. Figure 15 shows the 3D thermal field distribution and two orthogonal slices visualized by the surgical navigation software. The parameter values we used for simulation are listed in Table 2 (41).

Figure 16 gives the result of coagulation after $600 \mathrm{~s}$ with the MW power setting at $60 \mathrm{~W}$. Let $V_{S}$ and $V_{C}$ denote the volume surrounded by the isosurface of $54^{\circ} \mathrm{C}$ in the simulated thermal field and the actual experiment, respectively. Then, the relative error of thermal field simulation model can be defined as $\varepsilon=\left|V_{S}-V_{C}\right| / V_{C}$. The experiments were repeated 20 times and the mean value of the relative error was $5.6 \%$.

\section{Conclusions and Future Work}

In this study, a 3D US image-guided robotic system was developed for improved MW ablation of malignant liver 

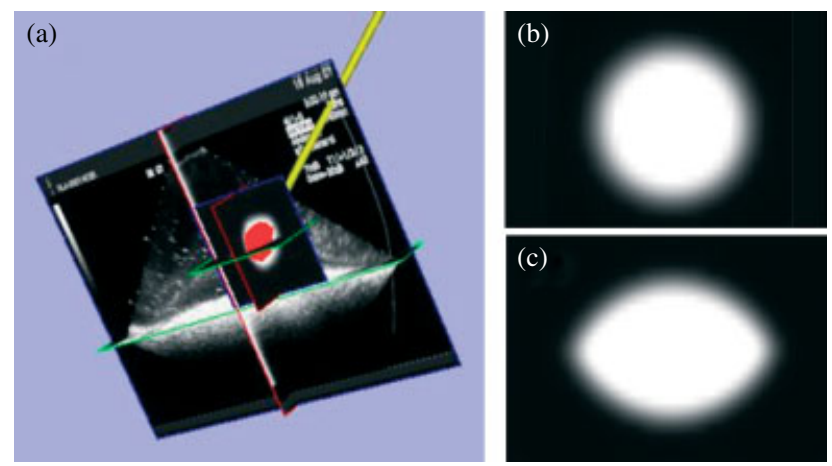

Figure 15. Simulated thermal field distribution: (a) 3D scene; (b) top view; (c) lateral view

Table 2. Physical properties of the biological tissues

\begin{tabular}{lcc}
\hline Property & Blood vessel & Liver tissue \\
\hline Density $\rho\left(\mathrm{kg} / \mathrm{m}^{3}\right)$ & 1040 & 1030 \\
Specific heat capacity c $(\mathrm{J} / \mathrm{kg} / \mathrm{k})$ & 3500 & 3600 \\
Thermal conductivity $k(\mathrm{~W} / \mathrm{m} / \mathrm{k})$ & 0.600 & 0.497 \\
Blood flow rate $F\left(\mathrm{~m}^{3} / \mathrm{kg} / \mathrm{s}\right)$ & 0.0 & $1.67 \times 10^{-5}$ \\
\hline
\end{tabular}

tumours. The robotic system includes four components: (a) a freehand-tracked 3D US imaging module to identify the target location; (b) the needle-driven robot to place the MW antenna into the target; (c) the MW thermal field simulation module to identify the MW power level and coagulation time for the MW ablative treatment; and (d) the surgical navigation software, which hosts the imaging, thermal field simulation and supervisory control of the needle-driven robot. Two types of wrists have been developed for our robotic system, viz. the RCM wrist and the non-RCM wrist. Precise needle placement and accurate estimation of the MW thermal field distribution, two critical requirements for accurate MW thermal coagulation, can be achieved by using our robotic system.

We tested the needle placement accuracy for a fixed and rigid target in the mechanical phantom experiment from an engineering perspective. The robotic system achieved an entry accuracy of $2.62 \pm 1.21 \mathrm{~mm}$ and a targeting accuracy of $2.94 \pm 1.34 \mathrm{~mm}$ when the RCM wrist was installed on the robot. When the non-RCM wrist was used, the entry and targeting accuracy for the robotic system was improved to $2.01 \pm 0.73$ and $2.63 \pm 1.20 \mathrm{~mm}$, respectively, due to the reduced transmission error of the non-RCM wrist compared with the RCM mechanism. If the positioning errors caused by the US imaging module were excluded, the entry and target errors introduced by the non-RCM robot alone were $1.17 \pm 0.82$ and $1.40 \pm 0.84 \mathrm{~mm}$, respectively. Another mechanical phantom indicated that the 3D US reconstruction errors along the $x, y$ and $z$ axes of the 3D template were 0.28 , 0.30 and $0.61 \mathrm{~mm}$, respectively. We see that the overall positioning error for the needle placement almost equals the summation of errors introduced by the US imaging module and the needle-driven robot.

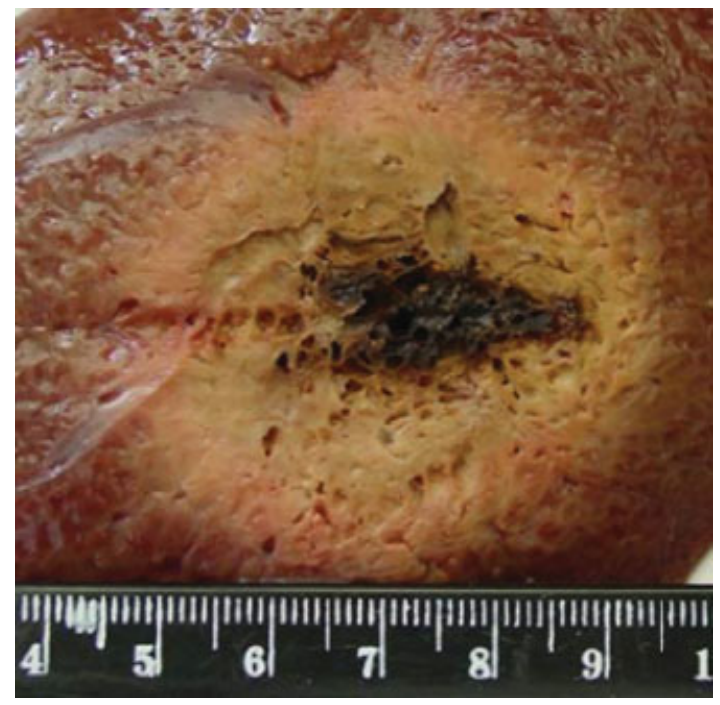

Figure 16. MW coagulation of the porcine liver

The needle targeting accuracy can be further improved if fine adjustment of the needle insertion according to the real-time 2D US image feedback through onscreen display is introduced. This fact was verified in the artificial-tissue phantom experiment and the needletargeting accuracy for the RCM robot was improved to $1.6 \pm 1.0 \mathrm{~mm}$. Our proposed slice guidance method, a qualitative technique based on the comparison of the preoperative and intraoperative model, could partially compensate for the impact of target motion. This was verified by our ex vivo experiment, in which the non-RCM robot achieved a targeting accuracy of $1.8 \pm 0.9 \mathrm{~mm}$; even the target motion was introduced. Clinical application of this slice guidance method is based on the fact that the patient could consciously regulate his breathing according to the physician's instructions. Our future plan for the target motion is to create a quantitative comparison metric for fine registration and real-time motion compensation $(6,17)$ via visual servo-control $(14)$.

In order to determine the MW power and coagulation time, two critical parameters for accurate MW ablative therapy, we developed a MW thermal field simulation model. The model was verified by an ex vivo experiment on porcine liver tissue, achieving a $5.6 \%$ relative mean error between the predicted and experimental coagulated necrosis volumes. In the future, intensive in vivo experiments will be required for further verification of the proposed thermal field model and construction of the clinical database.

Although originally designed for MW ablation of liver tumours, the robotic system can be reconfigured for other applications, such as the percutaneous puncture treatment of liver or kidney cysts, which shares the same needle placement procedure as the image-guided MW coagulation treatment. Our non-RCM robotic system has already been used to treat more than 20 patients with liver or kidney cysts. In all these experiments, the entry error for the needle placement was $<2 \mathrm{~mm}$. Therefore, our robotic system holds promise to enhance the clinical 
performance of percutaneous MW ablation of malignant liver tumours.

\section{Acknowledgements}

Most of the work presented in this paper was research conducted by the Robot and Automation Laboratory (RAL) at Tsinghua University, in collaboration with the Ultrasound Department at the Chinese PLA General Hospital (PLAGH). The authors would like to acknowledge Dr Dunmin Lu and graduate students Jing Xiong, Shaoli Liu, Senqiang Zhu, Tao Feng and Hongbo Wang from the RAL group. They would also like to thank Yang Wang, Tong $\mathrm{Lu}$ and He Ren from the PLAGH group. Financial support for this work was mainly provided by the National Natural Science Foundation of China (NSFC) under Grant No. 50675109 and the Beijing Municipal Science and Technology Commission (BMSTC) under Grant No. H060720050330.

\section{References}

1. World Cancer Research Fund/American Institute for Cancer Research (AICR). Food, Nutrition, Physical Activity, and the Prevention of Cancer: a Global Perspective. AICR: Washington, DC, 2007.

2. Dong BW, Liang P, Yu X, et al. Percutaneous sonographically guided microwave coagulation therapy for hepatocellular carcinoma: results in 234 patients. Am J Roentgenol 2003; 180: 1547-1555.

3. National Cancer Institute, Liver Cancer Homepage: http://www.cancer.gov/cancertopics/types/liver.

4. El-Serag H, Rudolph KL. Hepatocellular carcinoma: epidemiology and molecular carcinogenesis. Gastroenterology 2007; 132: 2557-2576.

5. Department of Pathology, Johns Hopkins University. The Liver Cancer web page: http://pathology2.jhu.edu/liver.

6. Boctor EM, Choti MA, Burdette EC, et al. Three-dimensional ultrasound-guided robotic needle placement: an experimenta evaluation. Int J Med Robotics Comput Assist Surg 2008; 4(2): 180-191.

7. Boctor EM, Fischer G, Choti M, et al. Dual-armed robotic system for intraoperative ultrasound guided hepatic ablative therapy: a prospective study. Proceedings of IEEE International Conference on Robotics and Automation, 2004; 377-382.

8. Liang P, Wang Y, Yu XL, et al. Malignant liver tumors: treatment with percutaneous microwave ablation - complications among cohort of 1136 patients. Radiology 2009; 251(3): 933-940.

9. Xu J, Song ZJ, Jia ZZ. A preoperative ultrasound planning of robotic system for hepatic microwave coagulation. Proceedings of IEEE International Conference on Automation and Logistics, 2009.

10. Fenster A, Downey DB, Cardinal NH. Three-dimensional ultrasound imaging. Phys Med Biol 2001; 46: 67-99.

11. Prager RW, Gee AH, Berman L. Stradx: real-time acquisition and visualization of freehand three-dimensional ultrasound. Med Image Anal 1999; 3(2): 129-140.

12. Prager RW. The Stradx 3D ultrasound acquisition and visualization system: http://mi.eng.cam.ac.uk/ rwp/stradx/.

13. 3D Slicer: http://www.slicer.org.

14. Abolmaesumi P, Salcudean SE, Zhu WH, et al. Image-guided control of a robot for medical ultrasound. IEEE Trans Robot Autom 2002; 18(1): 11-23.

15. Boctor EM, Webster RJ III, Mathieu H, et al. Virtual remote center of motion control for needle placement robots. Comput Aided Surg 2004; 9(5): 175-183.

16. Center for Medicine and Technology (MISON), Norway: http://www.mison.no.

17. Hong JS, Dohi T, Hashizume M, et al. A motion adaptable needle placement instrument based on tumor specific ultrasonic image segmentation. MICCAI-2002. Lect Notes Comput Sci 2002; 2488: 122-129.

18. Hong JS, Dohi T, Hashizume M, et al. An ultrasound-driven needle insertion robot for percutaneous cholecystostomy. Phys Med Biol 2004; 49(3): 441-455.

19. Tungjitkusolmun S, Staelin ST, Haemmerich D, et al. Threedimensional finite-element analyses for radio-frequency hepatic tumor ablation. IEEE Trans Biomed Eng 2002; 49(1): 3-9.

20. Chang IA, Nguyen UD. Thermal modeling of lesion growth with radiofrequency ablation devices. BioMed Eng OnLine 2004; 3: 27.

21. Liang P, Dong BW, Yu XL, et al. Computer-aided dynamic simulation of microwave-induced thermal distribution in coagulation of liver cancer. IEEE Trans Biomed Eng 2001; 48(7): $821-829$.

22. Ascension Technology Corporation. 6D BIRD CLASS B Position and Orientation Measurement System Installation and Operation Guide, April 2003.

23. Taylor RH, Stoianovici D. Medical robotics in computerintegrated surgery. IEEE Trans Robot Autom 2003; 19(5): 765-781.

24. Xu J. Research on the System Accuracy of the Ultrasoundguided Robot for Liver Cancer Coagulation Therapy. PhD Thesis, Department of Precision Instruments and Mechanology, Tsinghua University, Beijing, China, November 2007.

25. Gee AH, Prager RW, Treece GM, et al. Engineering a freehand 3D ultrasound system. Pattern Recogn Lett 2003; 24(4-5): 757-777.

26. Rohling RN, Gee AH, Berman L. A comparison of freehand threedimensional ultrasound reconstruction techniques. Med Image Anal 1999; 3(4): 339-359.

27. $\mathrm{Xu} \mathrm{J}$, Chen $\mathrm{K}$, Yang XD, et al. Adaptive level set method for segmentation of liver tumors in minimally invasive surgery using ultrasound images. The 1st International Conference on Bioinformatics and Biomedical Engineering (ICBBE), 2007; 1091-1094.

28. Pagoulatos N, Haynor DR, Kim Y. A fast calibration method for 3D tracking of ultrasound images using a spatial localizer. Ultrasound Med Biol 2001; 27: 1219-1229.

29. Arun KS, Huang TS, Blostein SD. Least-squares fitting of two 3D point sets. IEEE Trans Pattern Anal Mach Intel 1987; 9(5): 698-700.

30. Eggert DW, Lorusso A, Fisher RB. Estimating 3D rigid body transformations: a comparison of four major algorithms. Mach Vision Appl 1997; 9: 272-290.

31. CimCore, Infinite 2.0: http://www.cimcore.com/infinite-2-0-2.

32. Song MW, Hutchinson S, Vidyasagar M. Robot Modeling and Control. Wiley: New York, 2005.

33. Zhu L, Xu LX, Chencinski N. Quantification of the 3D electromagnetic power absorption rate in tissue during transurethral prostatic microwave thermotherapy using heat transfer model. IEEE Trans Biomed Eng 1998; 45(9): 1163-1172.

34. Besl PJ, McKay ND. A method for registration of 3D shapes. IEEE Trans Patt Anal Mach Intell 1992; 14(2): 239-256.

35. Rusinkiewicz S, Levoy M. Efficient variants of the ICP algorithm. Third International Conference on 3D Digital Imaging and Modeling (3DIM), 2001.

36. Kitware: visualization toolkit (VTK): http://www.vtk.org/.

37. Kitware: segmentation and registration toolkit (ITK): http://www.itk.org/.

38. Solberg OV, Lindseth $\mathrm{F}$, Torp $\mathrm{H}$, et al. Freehand 3D ultrasound reconstruction algorithms - a review. Ultrasound Med Biol 2007; 33(7): 991-1009.

39. Koizumi N, Lee D, Ota K, et al. A framework of the noninvasive ultrasound theragnostic system. Proceedings of the 4th International Workshop on Medical Imaging and Augmented Reality, 2008; 231-240.

40. Pennes HH. Analysis of tissue and arterial temperature in the resting human forearm. $J$ Appl Physiol 1948; 1: 93-122.

41. Van Den Berg PM, De Hoop AT, Segal A, et al. A computational model of the electromagnetic heating of biological tissue with application to hyperthermic cancer therapy. IEEE Trans Biomed Eng 1983; 30(12): 797-805. 